\section{Growth and Photosynthetic Capability of Momordica grosvenori Plantlets Grown Photoautotrophically in Response to Light Intensity}

\author{
Meijun Zhang \\ College of Life Science, Capital Normal University, No. 105 North \\ Xisanhuan Road, Beijing 100048, China; and Yangtze Delta Region \\ Institute of Tsinghua University, Zhejiang 314100, China
}

Duanduan Zhao, Zengqiang Ma, and Xuedong Li College of Life Science, Capital Normal University, Beijing 100048, China

Yulan Xiao ${ }^{1}$

College of Life Science, Capital Normal University, No. 105 North Xisanhuan Road, Beijing 100048, China; and Yangtze Delta Region Institute of Tsinghua University, Zhejiang 314100, China

Additional index words. micropropagation, callus formation, rooting, chlorophyll content, electron transport rate, light acclimation

\begin{abstract}
Momordica grosvenori plantlets were cultured in vitro for $26 \mathrm{~d}$ on sucrose- and hormone-free Murashige and Skoog (MS) medium with four levels of photosynthetic photon flux density $(P P F D)$, namely $25,50,100$, or $200 \mu \mathrm{mol} \cdot \mathrm{m}^{-2} \cdot \mathrm{s}^{-1}$, and a $\mathrm{CO}_{2}$ concentration of $1000 \mu \mathrm{mol} \cdot \mathrm{mol}^{-1}$ in the culture room [i.e., photoautotrophic micropropagation (PA) treatments]. The control treatment was a photomixotrophic culture using MS medium containing sucrose and NAA with a $\mathrm{CO}_{2}$ concentration of 400 $\mu \mathrm{mol} \cdot \mathrm{mol}^{-1}$ in the culture room and a PPFD of $25 \mu \mathrm{mol} \cdot \mathrm{m}^{-2} \cdot \mathrm{s}^{-1}$. Based on the results, a second experiment was conducted to investigate the effects of $\alpha$-naphthaleneacetic acid (NAA) and sucrose on callus formation. For this, plantlets were grown in the absence and presence of either NAA or sucrose. Compared with the control, the PA plantlet had a well-developed rooting system, better shoot, greater chlorophyll content, and higher electron transport rate and the ex vitro survival percentage was increased by $31 \%$. Both sucrose and NAA stimulated callus formation on the shoot bases of control plantlets, whereas calluses did not form on the plantlets grown in sucrose- and hormone-free medium. The stronger light intensities increased the fresh and dry weight of plantlets. A $P P F D$ of $100 \mu \mathrm{mol} \cdot \mathrm{m}^{-2} \cdot \mathrm{s}^{-1}$ was more suitable for the growth of $M$. grosvenori plantlets. Therefore, photoautotrophic plantlets grown at high light intensities would be better suited to the intense irradiance found in sunlight.
\end{abstract}

Momordica grosvenori Swingle or Lo Han Kuo (Chinese) is a perennial vine native to southern China in Guangxi province. The fruit has been used in traditional Chinese medicine to treat colds, coughs, sore throats, and gastrointestinal disorders. The fruit contains a natural sweetener called Mogroside V, which is 300 times sweeter than cane sugar and extremely low in calories (Cheng, 1987). Mogroside $\mathrm{V}$ has the potential to be more than just a beverage sweetener. Takasaki et al. (2003) and Yao et al. (2008) reported

\footnotetext{
Received for publication 4 Feb. 2009. Accepted for publication 6 Apr. 2009.

We are grateful for the financial support provided by the Science and Technology Department of Zhejiang Province, China. We are also thankful to Mr. Huabing Yan of Guangxi University for furnishing Momordica grosvenori plantlets used in this study.

${ }^{1}$ To whom reprint requests should be addressed; e-mailylxiao@yahoo.com.
}

that Mogroside V suppressed carcinogenesis in mice and reduces oxidative stress in the body.

Momordica grosvenori propagated by layering, grafting, and dividing of tuberous roots often have low multiplication rates and cause a variety of degeneration. These methods also result in low yields of fruits. Variety sue-cultured plantlets. These plantlets are conventionally propagated on sugar-containing medium with growth regulators, especially $\alpha$-naphthaleneacetic acid (NAA) added to the rooting medium. Tissue-cultured plantlets use the sugar in the medium as a carbon source, high light intensity is usually not necessary, and small airtight culture vessels must be used to avoid contamination. Lin et al. (2003) and Xu et al. (2006) reported problems associated with micropropagation of $M$. grosvenori. These problems included slow growth, low survival rates during acclimatization, and nonbearing or small fruits degeneration has been altered by using tis- during field production. The losses during acclimatization accounted for $20 \%$ to $50 \%$ of the plantlets ex vitro.

Photoautotrophic micropropagation (PA), using a sugar-free medium and leafy explant, in which plantlets use $\mathrm{CO}_{2}$ in the air as the sole carbon source, has several advantages over photomixotrophic micropropagation (PM). The advantages include minimal microbial contamination, increased photosynthesis, growth and rooting in vitro, and survival percentages ex vitro when the in vitro environment is controlled to maximize photosynthesis (Aitken-Christie et al., 1995; Kozai, 1991). High photosynthetic photon flux density $(P P F D)$ and a high $\mathrm{CO}_{2}$ concentration in the culture vessel significantly enhance photosynthesis of plantlets in vitro (Fujiwara et al., 1988; Kubota and Kozai, 2001). A high $\mathrm{CO}_{2}$ level in the vessel can be achieved by increasing $\mathrm{CO}_{2}$ concentration in the culture room and the number of air exchanges in the vessel. The latter, defined as the hourly vessel ventilation rate divided by the air volume of the vessel (Kozai et al., 1986), can be increased or decreased by changing the gaspermeable filter disks attached to the holes in the lid and walls of the vessel. In addition, photoautotrophic conditions would promote growth and rooting of the plantlets without growth regulators in the medium (Nguyen et al., 1999; Xiao and Kozai, 2004).

Practitioners of photoautotrophic culture have investigated the effects of light intensity on the development of various plant species. These include in vitro plantlets of Solanum tuberosum (Kitaya et al., 1995), Cymbidium (Kozai et al., 1990), and Gardenia jasminoides (Serret et al., 1996). These reports showed that light intensity affected the photosynthetic characteristics of plantlets in vitro. However, there are no reports about the effects of light intensity on growth and photosynthetic capability of $M$. grosvenori plantlets. Various plant species in nature show a number of acclimatization responses to light intensity (Anderson et al., 1995). For example, light intensity changes the number of chloroplasts and leaf thickness (Bailey et al., 2001) and chlorophyll content (Hirashima et al., 2006; Lindahl et al., 1995). The responses of photoautotrophic plantlets in vitro to high levels of irradiation may be similar to plants in a natural environment.

The objectives of this study were to: 1) produce $M$. grosvenori plantlets of good quality and high survival percentages by comparing plantlets grown under photomixotrophic and photoautotrophic conditions; and 2) investigate the effects of light intensity on growth, photosynthetic ability, and light acclimation of the photoautotrophic plantlets.

\section{Materials and Methods}

Plant material, treatments, and culture conditions. Single shoots, each with two unfolded leaves excised from in vitro cultures of $M$. grosvenori, were used as explants and cultured for $26 \mathrm{~d}$. The average leaf area, fresh 
and dry weight, and stem length per shoot was $602 \pm 47 \mathrm{~mm}^{2}, 98.2 \pm 11.3 \mathrm{mg}, 8.4 \pm 1.4 \mathrm{mg}$, and $2.1 \pm 0.3 \mathrm{~cm}$, respectively. These in vitro cultures of $M$. grosvenori had been cultured on hormone-free Murashige and Skoog (MS) (1962) medium containing $30 \mathrm{~g} \cdot \mathrm{L}^{-1}$ sucrose for three subcultures ( 5 weeks for each subculture) to remove the exogenous hormone accumulation before experiments. Four explants were cultured in each Magenta-type vessel (370 mL; Verde Co., Ltd., Toyohashi, Japan) containing $70 \mathrm{~mL}$ MS medium with the $\mathrm{pH}$ adjusted to 5.8 before autoclaving. Gelrite $^{\circledR}$ (Sigma G1910; Sigma, St. Louis, MO) was used as the gelling agent $\left(3.2 \mathrm{~g} \cdot \mathrm{L}^{-1}\right)$ because it was more transparent than agar and allowed visualization of root formation.

Expt. 1. The first experiment included four PA treatments, $\mathrm{L}_{25}, \mathrm{~L}_{50}, \mathrm{~L}_{100}$, and $\mathrm{L}_{200}$, in which $\mathrm{L}$ indicates light intensity and subscripts denote $P P F D$, and one PM treatment as a control at $25 \mu \mathrm{mol} \cdot \mathrm{m}^{-2} \cdot \mathrm{s}^{-1}$. Light intensity was measured on the surface of the empty shelves with a light meter (Model LI-250A; LI-COR, Lincoln, NE).

The PA explants were cultured on hormone- and sucrose-free MS medium, and $\mathrm{CO}_{2}$ concentration was maintained at 1000 $\mu \mathrm{mol} \cdot \mathrm{mol}^{-1}$ in the culture room. The $\mathrm{CO}_{2}$ concentrations were measured and controlled by a $\mathrm{CO}_{2}$ controller with a nondispersive infrared detector $\mathrm{CO}_{2}$ sensor (T6004; Telaire Co., Ltd., Shoreview, MN). The number of air exchanges of the culture vessels measured by the method of Kozai et al. (1986) were increased with culture time by increasing the area of gas-permeable filter disks $(10 \mathrm{~mm}$ in diameter, pore size $0.5 \mathrm{~mm}$; Milli-Seal, Nihon Millipore, Ltd., Tokyo, Japan) attached to the holes in the lid and walls of the vessel. To get a feasible ventilation rate for photosynthesis, the number of air exchanges of the culture vessels were 0.2 $\mathrm{h}^{-1}$ from Days 0 to $3,1.8 \mathrm{~h}^{-1}$ from Days 4 to 7 , $2.7 \mathrm{~h}^{-1}$ from Days 8 to 17 , and $3.8 \mathrm{~h}^{-1}$ from Days 18 to 25 .

The PM or control explants were cultured on sucrose-containing $\left(30 \mathrm{~g} \cdot \mathrm{L}^{-1}\right) \mathrm{MS}$ medium with $2.7 \mu \mathrm{M}$ NAA at a $\mathrm{CO}_{2}$ concentration of $400 \mu \mathrm{mol} \cdot \mathrm{mol}^{-1}$ in the culture room and a number of air exchanges of $0.2 \mathrm{~h}^{-1}$. The control treatment was very similar to conventional micropropagation of $M$. grosvenori plantlets.

The air temperature and relative humidity in the culture rooms were maintained at $25 \pm$ $1{ }^{\circ} \mathrm{C}$ and $80 \% \pm 5$, respectively. The photoperiod was $12 \mathrm{~h}$ per day supplied with coolwhite fluorescent lamps. Twelve replications (12 vessels) were used for each treatment. The experiment was conducted twice.

Expt. 2. Based on the results of the first experiment, an experiment was conducted to investigate the effects of sucrose and NAA on callus formation. This experiment included two PM and two PA treatments at 25 $\mu \mathrm{mol} \cdot \mathrm{m}^{-2} \cdot \mathrm{s}^{-1}$ light. The PM plantlets were cultured on sucrose-containing $\left(30 \mathrm{~g} \cdot \mathrm{L}^{-1}\right)$ medium with $2.7 \mu \mathrm{M}$ NAA (PMN treatment) or without NAA (PM treatment). The PA plantlets were cultured on sucrose-free medium with $2.7 \mu \mathrm{M}$ NAA (PAN treatment) or without NAA (PA treatment). The plant material, culture conditions, and treatment replications were the same as the first experiment for the PM and PA treatments, respectively. The experiment was conducted once. Chlorophyll content, the ratio of variable to maximum chlorophyll fluorescence $\left(\mathrm{F}_{\mathrm{v}} / \mathrm{F}_{\mathrm{m}}\right)$, and electron transport rate (ETR) were not measured in the second experiment.

Acclimation ex vitro and survival percentage. After $26 \mathrm{~d}$ of in vitro culture, plantlet growth was determined in half of the plantlets from each treatment of both experiments. The remaining plantlets were taken out from the vessels and washed to remove any medium adhering to the root system. The washed plantlets were transplanted into a tray with peat (Growing Mix I; Fafard Co. Ltd., Inkerman, Canada) and placed in a greenhouse with a simple shading screen for acclimatization. During acclimatization, relative humidity in the first week was kept at $80 \%$ to $90 \%$ with an automatic sprayer and then decreased gradually to $80 \%$ to $70 \%$ in the second week and $70 \%$ to $60 \%$ for the next days. The average air temperature was $22{ }^{\circ} \mathrm{C}$ day and $17{ }^{\circ} \mathrm{C}$ night. Twenty days after transplanting, the dead plantlets were counted in each treatment, and the survival percentages were determined.

Measurements, calculation, and statistical analysis. Rooting percentage was recorded every day until Day 16. Number of roots, stem and root length, fresh and dry weight of root and shoot, and leaf area were measured on Day 26. Leaf area was determined using image and quantify analysis software (LIA32 for Windows 95; K. Yamamoto, Nagoya, Japan) with an image scanner. Dry weight of the plantlets was determined after drying at $80{ }^{\circ} \mathrm{C}$ for $72 \mathrm{~h}$.

Chlorophyll content was measured after cutting pieces of the second and third fully expanded leaves (counted down from apex), soaking in $5 \mathrm{~mL} \mathrm{~N}, \mathrm{~N}$-dimethylformamide solution, and shaking once every $10 \mathrm{~min}$ for $2 \mathrm{~h}$ at $4{ }^{\circ} \mathrm{C}$ (Moran and Porath, 1980). Absorbance was measured with an ultraviolet-vis spectrophotometer (Helios Gamma; Thermo Spectronic Co., Ltd., Cambridge, U.K.) at wavelengths of $664 \mathrm{~nm}, 647 \mathrm{~nm}$, and 603 $\mathrm{nm}$. The chlorophyll content, including chlorophyll a and chlorophyll $\mathrm{b}$, was determined on a fresh weight basis $\left(\mu \mathrm{g} \cdot \mathrm{g}^{-1}\right)$ and calculated using the formula of Moran (1982).

The $\mathrm{F}_{\mathrm{v}} / \mathrm{F}_{\mathrm{m}}$ and ETR were measured and calculated with a Maxi-Imaging-PAM chlorophyll fluorescence measuring system (Walz, Effeltrich, Germany). After $24 \mathrm{~d}$ of culture, the second and third fully expanded leaves (counted down from apex) were used for the measurement. Six replications or six leaves were measured in each treatment. For chlorophyll fluorescence measurement, the plantlets were placed in a dark room to adapt for $15 \mathrm{~min}$. Next, randomly chosen intact leaves from five treatments were placed in the measuring head $(10 \times 13 \mathrm{~cm})$ to measure the $\mathrm{F}_{\mathrm{v}} / \mathrm{F}_{\mathrm{m}}$. The leaves without dark adaptation were used to evaluate ETRs. Each ETR value was obtained using actinic irradiance for $10 \mathrm{~s}$. All relevant fluorescence parameters, including actinic irradiance and leaf temperature, were recorded and ETRs were calculated in the Maxi-Imaging-PAM system.

Statistical significance was determined using Sigma Stat (SigmaStat ${ }^{\top M}$ for Windows V2.03; SPSS Inc., Chicago, IL). Differences between means were assessed with the HolmSidak test and a significance level of $P \leq 0.01$.

\section{Results}

\section{Expt. 1}

Root growth. The pattern of the root system in control and PA treatments differed significantly. Large callus mass formed on the shoot bases of control plantlets, whereas callus was not found on the shoot bases of PA plantlets (Fig. 1). In the control treatment, callus formation was observed on the shoot base on Day 3, callus volume then enlarged, and the roots emerged on Day 8. In contrast, roots in all PA treatments were formed directly from plantlets without an intermediate callus phase. The control plantlets had the highest number of main adventitious roots (Table 1) but were accompanied by large masses of callus and produced sparse lateral branching. The roots of control plantlets were firmly encased in callus and therefore difficult to separate. Accordingly, we determined the fresh and dry weights of roots that were outside of callus. On the other hand, the main adventitious root of the PA plantlet gave rise to a denser growth of fine lateral roots. This was especially true where the $\mathrm{L}_{100}$ treatment for the length and dry weights of the roots were 6.5 and 1.6 times greater than those in the control treatment, respectively.

Light intensity affected root formation. Rooting percentage reached $100 \%$ for all plantlets by Day 16 (Fig. 2). However, rooting occurred first in the $\mathrm{L}_{25}$ treatment on Day 7 and last in the $\mathrm{L}_{200}$ treatment on Day 10 . Rooting percentage reached $100 \%$ in the $\mathrm{L}_{25}$ treatment on Day 11, which was $5 \mathrm{~d}$ earlier than the $\mathrm{L}_{200}$ treatment. Root growth was greatest in the $\mathrm{L}_{100}$ treatment, in which the fresh weight of roots was 3.4 times greater than that in the $\mathrm{L}_{200}$ treatment, and the dry weight was 2.5 times greater than that in the $\mathrm{L}_{25}$ treatment (Table 1). Stronger light intensities also increased the ratio of root dry weight to root fresh weight $(\mathrm{DW} / \mathrm{FW})$ in PA-treated plantlets.

Shoot growth. PA treatments improved shoot growth over the control treatment (Table 2; Fig. 1). Leaf area, stem length, and fresh and dry weight of shoots were greatest in the $\mathrm{L}_{100}$ treatment, which were $2.7,1.2,3.0$, and 3.7 times greater, respectively, than those of control plantlets, but there was no significant difference in dry weight between the $\mathrm{L}_{200}$ and $\mathrm{L}_{100}$ treatments. Leaf area as well as fresh and dry weights of shoots were, respectively, 2.0, 1.8, and 1.8 times higher in PA-treated plantlets compared with the control plantlets grown under the same low light intensity (25 $\left.\mu \mathrm{mol} \cdot \mathrm{m}^{-2} \cdot \mathrm{s}^{-1}\right)$. 


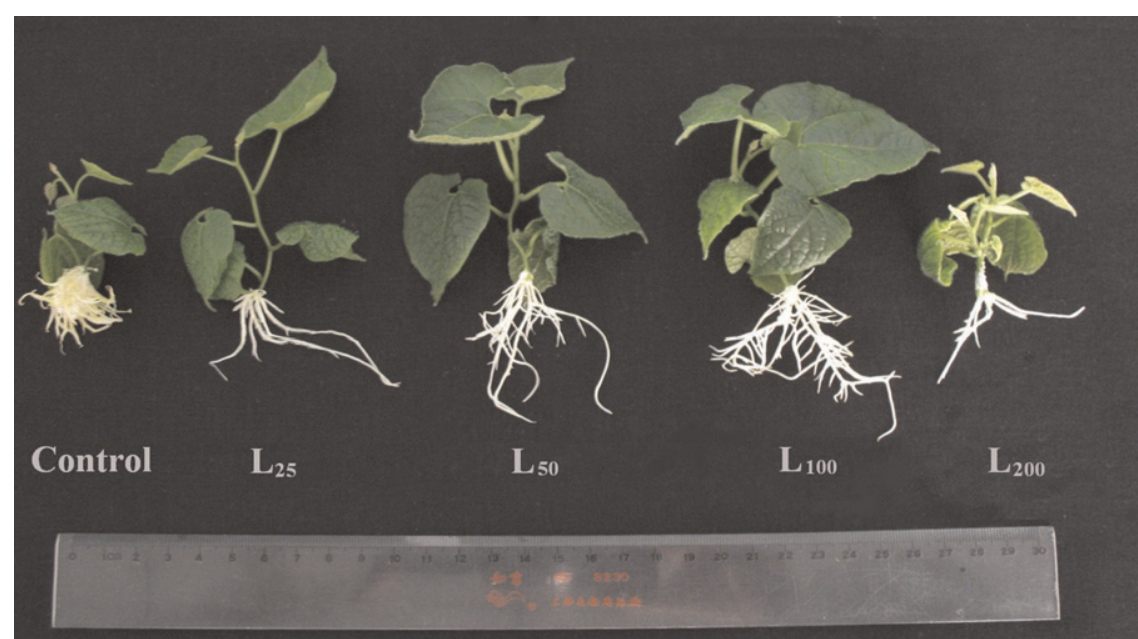

Fig. 1. Growth of Momordica grosvenori plantlets cultured on sucrose- and $\alpha$-naphthaleneacetic acid (NAA)-free medium with four levels of light intensity, $25,50,100$, or $200 \mu \mathrm{mol} \cdot \mathrm{m}^{-2} \cdot \mathrm{s}^{-1}$ for $26 \mathrm{~d}$. $\mathrm{L}_{25}$, $\mathrm{L}_{50}, \mathrm{~L}_{100}$, and $\mathrm{L}_{200}$ were four photoautotrophic treatments, where $\mathrm{L}$ indicates light intensity and subscripts denote photosynthetic photon flux density (PPFD). The control was a photomixotrophic treatment using sucrose-containing medium with $2.7 \mu \mathrm{M} \mathrm{NAA}$ at a $P P F D$ of $25 \mu \mathrm{mol} \cdot \mathrm{m}^{-2} \cdot \mathrm{s}^{-1}$.

Among all PA treatments, growth was improved as the light intensity increased from $\mathrm{L}_{25}$ to $\mathrm{L}_{100}$, but the $\mathrm{L}_{200}$ treatment had the lowest leaf area and stem length (but not lowest dry weight). Stronger light intensities also increased shoot DW/FW and leaf thickness and produced longer and denser epidermal hairs in all PA-treated plantlets. The branch, thickset stem, and small, thick, and yellow leaves of the plantlets were observed in the $\mathrm{L}_{200}$ treatment (Fig. 1).

Chlorophyll content and chlorophyll $a / b$ ratio. The chlorophyll content of plantlets was significantly influenced by the different culture methods (PA or PM) (Fig. 3). Among all the treatments, the plantlet chlorophyll content was greatest in the $\mathrm{L}_{50}$ treatment, being 2.1 times higher than that of the control. PA-treated plantlets $\left(\mathrm{L}_{25}\right.$ treatment) had 1.6 times more chlorophyll content than did the control even at the same low light intensity $\left(25 \mu \mathrm{mol} \cdot \mathrm{m}^{-2} \cdot \mathrm{s}^{-1}\right)$.

Light intensity also had a significant influence on chlorophyll content. The chlorophyll content increased to a maximum as the light intensified to $50 \mu \mathrm{mol} \cdot \mathrm{m}^{-2} \cdot \mathrm{s}^{-1}$ and then decreased to a minimum at $200 \mu \mathrm{mol} \cdot \mathrm{m}^{-2} \cdot \mathrm{s}^{-1}$, creating a parabola (Fig. 3). In contrast, the ratio of chlorophyll a to chlorophyll b increased consistently as the light intensified.
Ratio of variable to maximum chlorophyll fluorescence ratio and electron transport rate. The $\mathrm{F}_{\mathrm{v}} / \mathrm{F}_{\mathrm{m}}$ ratio, reflecting the maximal photochemical efficiency of photosystem II (PSII), is usually steady with fluctuations from 0.75 to 0.85 for nonstressed plants (Bolhar-Nordenkampf et al., 1989). In the present study, the $\mathrm{F}_{\mathrm{v}} / \mathrm{F}_{\mathrm{m}}$ (determined using the second or third leaf from the apex) ranged from 0.78 to 0.82 except for the $\mathrm{L}_{200}$, which had a ratio of 0.57 in the second leaf (data not shown). This suggests that photoinhibition had probably occurred in the second leaf taken from the $\mathrm{L}_{200}$ treatment, and consequently, it had the lowest maximum ETR (Fig. 4A).

ETRs allow for the rapid and noninvasive assessment of the light response of PSII and are used to study the photosynthetic performance and photoacclimation of photosynthetic organisms (Serôdio et al., 2006). The maximum ETR was related to the maximum photosynthetic capability, which was obtained when the rate of photosynthesis was limited by the activity of the electron transport chain or Calvin cycle enzymes (Behrenfeld et al., 2004; Ralph et al., 2005). Except for the second leaf from the $\mathrm{L}_{200}$ treatment, all the ETRs in the PA plantlets were higher than those of the control plantlets, and greater

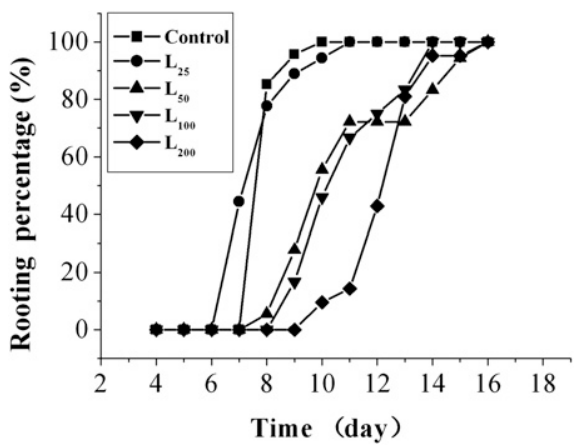

Fig. 2. Effects of four levels of light intensity, $25,50,100$, or $200 \mu \mathrm{mol} \cdot \mathrm{m}^{-2} \cdot \mathrm{s}^{-1}$ on the rooting percentage of Momordica grosvenori plantlets. $\mathrm{L}_{25}, \mathrm{~L}_{50}, \mathrm{~L}_{100}$, and $\mathrm{L}_{200}$ were four photoautotrophic treatments using sucrose- and $\alpha$ naphthaleneacetic acid (NAA)-free medium, where $\mathrm{L}$ indicates light intensity and subscripts denote photosynthetic photon flux density $(P P F D)$. The control was a photomixotrophic treatment using sucrose-containing medium with $2.7 \mu \mathrm{M}$ NAA at a PPFD of 25 $\mu \mathrm{mol} \cdot \mathrm{m}^{-2} \cdot \mathrm{s}^{-1}$.

light intensity increased the maximum ETRs of the PA plantlets (Fig. 4).

Survival ex vitro. In the present study, survival percentage was $65 \%$ lowest in the control treatment and 100\% highest in the $\mathrm{L}_{100}$ and $\mathrm{L}_{200}$ treatments (Table 2). The control plantlets, especially the smaller ones, wilted immediately when placed in the greenhouse. Approximately $85 \%$ of the wilted plantlets recovered, but some died in the middle of acclimatization as a result of root decay. In contrast, the PA plantlets wilted very little during the first day of acclimatization, recovered the next morning, and had over 95\% survival on Day 20.

\section{Expt. 2}

Effects of sucrose and $\alpha$-naphthaleneacetic acid on callus formation. Calluses only formed on the shoot base of PM plantlet. The difference between the PM and PA treatments was that the PM plantlets used sucrose as a carbon source with $2.7 \mu \mathrm{M}$ NAA in the medium, but the PA plantlets used $\mathrm{CO}_{2}$ as a carbon source without NAA in the medium.

The results from the second experiment showed that both sucrose and NAA stimulated callus formation (Table 3; Fig. 5). The callus texture was more compact in the PMN

Table 1. Effects of four levels of light intensity on rooting percentage and root growth of Momordica grosvenori per plantlet cultured on sucrose-free medium without NAA for $26 \mathrm{~d}$.

\begin{tabular}{|c|c|c|c|c|c|c|}
\hline \multirow[b]{2}{*}{ Treatment } & \multirow[b]{2}{*}{ Rooting (\%) } & \multirow[b]{2}{*}{ Number of roots } & \multirow[b]{2}{*}{ Root length $(\mathrm{cm})$} & \multicolumn{3}{|c|}{ Root wt (mg) } \\
\hline & & & & Fresh & Dry & $\mathrm{DW} / \mathrm{FW}(\%)$ \\
\hline Control & 100 & $22.8 \pm 4.6 \mathrm{a}^{\mathrm{y}}$ & $1.2 \pm 0.3 \mathrm{c}$ & $148.6 \pm 19.2 b$ & $5.2 \pm 0.6 \mathrm{~b}$ & 3.5 \\
\hline${ }^{\mathrm{z}} \mathrm{L}_{25}$ & 100 & $2.5 \pm 0.3 \mathrm{c}$ & $6.7 \pm 0.6 \mathrm{a}$ & $81.8 \pm 7.9 \mathrm{c}$ & $3.3 \pm 0.4 \mathrm{c}$ & 4.0 \\
\hline $\mathrm{L}_{50}$ & 100 & $4.1 \pm 0.4 \mathrm{bc}$ & $7.3 \pm 0.5 \mathrm{a}$ & $138.5 \pm 13.1 \mathrm{~b}$ & $5.5 \pm 0.6 b$ & 4.1 \\
\hline $\mathrm{L}_{100}$ & 100 & $4.4 \pm 0.4 b$ & $7.8 \pm 0.5 \mathrm{a}$ & $187.3 \pm 12.3 \mathrm{a}$ & $8.3 \pm 0.7 \mathrm{a}$ & 4.4 \\
\hline $\mathrm{L}_{200}$ & 100 & $5.0 \pm 0.6 \mathrm{~b}$ & $3.3 \pm 0.3 \mathrm{~b}$ & $55.0 \pm 6.8 \mathrm{c}$ & $4.3 \pm 0.5 \mathrm{bc}$ & 12.3 \\
\hline
\end{tabular}

${ }^{\mathrm{z}} \mathrm{L}_{25}, \mathrm{~L}_{50}, \mathrm{~L}_{100}$, and $\mathrm{L}_{200}$ were four photoautotrophic treatments, where $\mathrm{L}$ indicates light intensity and subscripts denote photosynthetic photon flux density $(P P F D), 25,50,100$, or $200 \mu \mathrm{mol} \cdot \mathrm{m}^{-2} \cdot \mathrm{s}^{-1}$, respectively. The control was a photomixotrophic treatment using sucrose-containing medium with $2.7 \mu \mathrm{M} \alpha-$ naphthaleneacetic acid (NAA) at a $P P F D$ of $25 \mu \mathrm{mol} \cdot \mathrm{m}^{-2} \cdot \mathrm{s}^{-1}$.

${ }^{\mathrm{y}}$ Data are mean $\pm \mathrm{SE}$, and treatments denoted by the same letter in a column were not significantly different $(P \leq 0.01)$

$\mathrm{DW} / \mathrm{FW}=$ dry weight/fresh weight. 
Table 2. Effects of four levels of light intensity on shoot growth of Momordica grosvenori per plantlet cultured on sucrose-free medium without NAA for $26 \mathrm{~d}$ and survival percentage ex vitro on Day 20.

\begin{tabular}{lccrrr}
\hline & & & \multicolumn{3}{c}{ Shoot wt $(\mathrm{mg})$} \\
\cline { 3 - 6 } Treatment & Leaf area $\left(\mathrm{mm}^{2}\right)$ & Stem length $(\mathrm{cm})$ & Fresh & Dry & DW/FW $(\%)$ \\
\hline Control & $1,412 \pm 76 \mathrm{c}^{\mathrm{y}}$ & $4.0 \pm 0.2 \mathrm{ab}$ & $281.0 \pm 15.1 \mathrm{c}$ & $26.2 \pm 2.5 \mathrm{~d}$ & 9.3 \\
${ }^{\mathrm{z}} \mathrm{L}_{25}$ & $2,802 \pm 228 \mathrm{~b}$ & $4.0 \pm 0.2 \mathrm{ab}$ & $499.4 \pm 27.0 \mathrm{~b}$ & $48.3 \pm 2.8 \mathrm{c}$ & 9.7 \\
$\mathrm{~L}_{50}$ & $3,650 \pm 232 \mathrm{a}$ & $4.5 \pm 0.2 \mathrm{a}$ & $751.0 \pm 53.5 \mathrm{a}$ & $80.1 \pm 6.9 \mathrm{~b}$ & 10.6 \\
$\mathrm{~L}_{100}$ & $3,740 \pm 270 \mathrm{a}$ & $4.7 \pm 0.2 \mathrm{a}$ & $846.8 \pm 49.0 \mathrm{a}$ & $97.1 \pm 5.6 \mathrm{a}$ & 11.5 \\
$\mathrm{~L}_{200}$ & $2,198 \pm 121 \mathrm{~b}$ & $3.6 \pm 0.2 \mathrm{~b}$ & $612.9 \pm 34.7 \mathrm{~b}$ & $100.7 \pm 4.1 \mathrm{a}$ & 96 \\
\hline
\end{tabular}

${ }^{2} \mathrm{~L}_{25}, \mathrm{~L}_{50}, \mathrm{~L}_{100}$, and $\mathrm{L}_{200}$ were four photoautotrophic treatments, where $\mathrm{L}$ indicates light intensity and subscripts denote photosynthetic photon flux density $(P P F D), 25,50,100$, or $200 \mu \mathrm{mol} \cdot \mathrm{m}^{-2} \cdot \mathrm{s}^{-1}$, respectively. The control was a photomixotrophic treatment using sucrose-containing medium with $2.7 \mu \mathrm{M} \alpha-$ naphthaleneacetic acid (NAA) at a $P P F D$ of $25 \mu \mathrm{mol} \cdot \mathrm{m}^{-2} \cdot \mathrm{s}^{-1}$.

${ }^{\mathrm{y}} \mathrm{Data}$ are mean $\pm \mathrm{SE}$, and treatments denoted by the same letter in a column were not significantly different $(P \leq 0.01)$.

$\mathrm{DW} / \mathrm{FW}=$ dry weight/fresh weight.

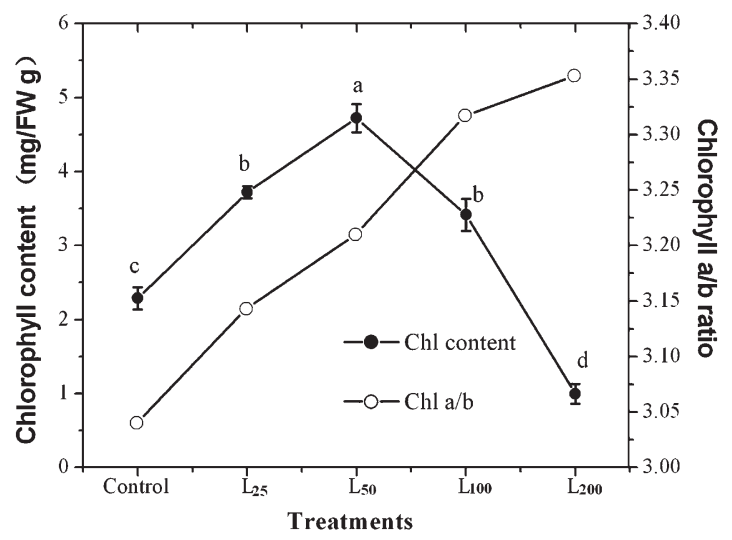

Fig. 3. Effects of four levels of light intensity, $25,50,100$, or $200 \mu \mathrm{mol} \cdot \mathrm{m}^{-2} \cdot \mathrm{s}^{-1}$ on the chlorophyll content and chlorophyll $\mathrm{a} / \mathrm{b}$ ratio of Momordica grosvenori plantlets. $\mathrm{L}_{25}, \mathrm{~L}_{50}, \mathrm{~L}_{100}$, and $\mathrm{L}_{200}$ were four photoautotrophic treatments using sucrose- and $\alpha$-naphthaleneacetic acid (NAA)-free medium, where $\mathrm{L}$ indicates light intensity and subscripts denote photosynthetic photon flux density $(P P F D)$. The control was a photomixotrophic treatment using sucrose-containing medium with $2.7 \mu \mathrm{M}$ NAA at a $P P F D$ of $25 \mu \mathrm{mol} \cdot \mathrm{m}^{-2} \cdot \mathrm{s}^{-1}$. Vertical bars represent SE.

and PAN treatments with NAA than it was in the PM treatment without NAA. The volume and dry weight of the callus and the number of roots were largest or highest in the PMN treatment followed by the PAN treatment (Table 3). The addition of NAA shortened the root length and produced sparse lateral branching.

Compared with the treatments with NAA, the PM treatment produced smaller callus masses and fewer adventitious roots with little lateral branching. The plantlets grown on sucrose- and NAA-free medium had no calluses and produced adventitious roots with well-developed lateral branching. Although the dry weight of the roots in the PA treatment was lower than that in the PMN treatment, the high survival percentage with PA indicates that the root system was more vigorous. The shoot dry weight was approximately two times greater in the PA and PAN treatments than that in the PM and PMN treatments.

\section{Discussion}

Rooting and callus formation. The plantlets cultured on sucrose- and NAA-containing medium produced more short roots but had large callus masses at the shoot bases. The results of the second experiment suggest that NAA and, a lesser extent, sucrose caused callus formation and influenced the number of roots. NAA with the presence or absence of sucrose in the medium stimulated callus formation and produced more adventitious roots with less lateral branching. The same was seen for plantlets grown in medium containing sucrose with or without NAA. Plantlets grown on sucrose- and NAAfree medium had no callus and produced more vigorous adventitious roots with welldeveloped lateral branching. Clearly, sucrose and NAA were unnecessary for root growth of $M$. grosvenori under photoautotrophic conditions. We therefore suggest that photoautotrophically grown plantlets synthesize appropriate endogenous growthpromoting hormones. Xiao and Kozai (2004) argued that endogenous phytohormones such as auxin, which are necessary for rooting and protein synthesis, are produced more by plantlets grown photoautotrophically than by plantlets grown photomixotrophically.

Light intensity influenced growth and development of the roots. The stronger the light intensity, the later the adventitious roots formed in all PA treatments (Fig. 1). Yellow or wilted leaves were observed in higher light-treated plantlets at the beginning of the culture period. The higher light intensities probably increased the temperature inside the vessel, which promoted transpiration in the
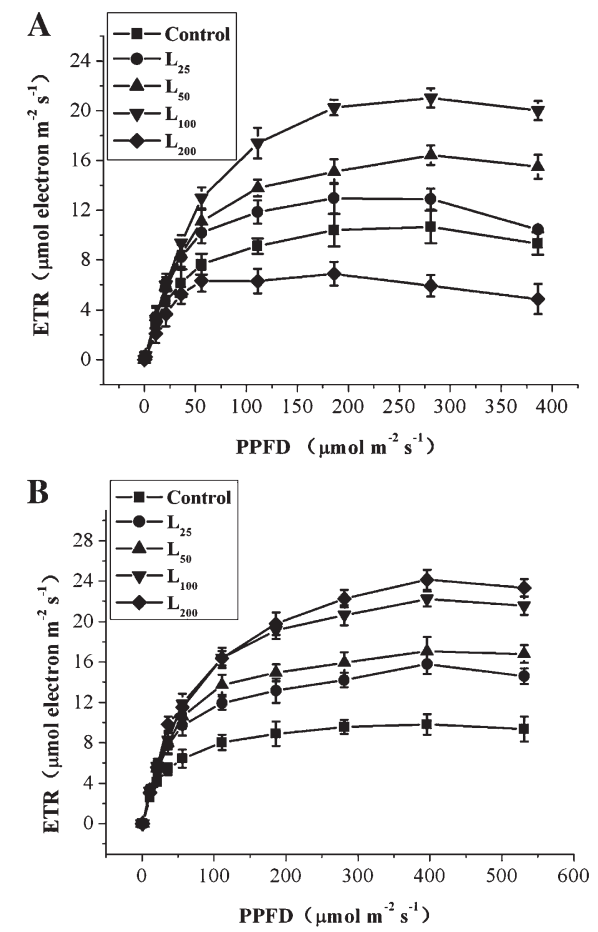

Fig. 4. The rapid light curves of Momordica grosvenori plantlets at four levels of light intensity, $25,50,100$, or $200 \mu \mathrm{mol} \cdot \mathrm{m}^{-2} \cdot \mathrm{s}^{-1}$ on Day 24. $\mathrm{L}_{25}, \mathrm{~L}_{50}, \mathrm{~L}_{100}$, and $\mathrm{L}_{200}$ were four photoautotrophic treatments, where $\mathrm{L}$ indicates light intensity and subscripts denote photosynthetic photon flux density (PPFD). The control was a photomixotrophic treatment using sucrose-containing medium with $2.7 \mu \mathrm{M}$ $\alpha$-naphthaleneacetic acid at a PPFD of 25 $\mu \mathrm{mol} \cdot \mathrm{m}^{-2} \cdot \mathrm{s}^{-1}$ (A) for the second leaf and (B) for the third from the apex of the shoot. Vertical bars represent SE $(n=6)$.

plantlets and caused water loss from the leaves. Thus, the higher light-treated explants needed a relatively longer period to recover, resulting in delay of root formation. In addition, root formation in plantlets is dependent on the auxin concentration. Light intensity probably affected synthesis and transport of indole-3-acetic acid (IAA) within the plantlets, which consequently influenced root formation. However, the relationship between light intensity and auxin synthesis is not known and additional study is justified.

Photosynthesis and chlorophyll content. The slow growth of plantlets has frequently 
Table 3. Effects of sucrose and NAA on growth of Momordica grosvenori plantlets on Day 26 and survival percentage ex vitro on Day 20.

\begin{tabular}{|c|c|c|c|c|c|c|c|}
\hline \multirow[b]{2}{*}{ Treatment } & \multicolumn{2}{|c|}{ Callus } & \multicolumn{3}{|c|}{ Root } & \multirow{2}{*}{$\frac{\text { Shoot }}{\text { DW (mg) }}$} & \multirow[b]{2}{*}{ Survival ex vitro $(\%)$} \\
\hline & Volume $\left(\mathrm{mm}^{3}\right)$ & DW (mg) & Number & Length $(\mathrm{cm})$ & DW (mg) & & \\
\hline$\overline{\mathrm{PMN}^{\mathrm{z}}}$ & $1,300 \pm 200 a^{y}$ & $40.2 \pm 5.2 \mathrm{a}$ & $20.6 \pm 5.6 \mathrm{a}$ & $1.2 \pm 0.5 \mathrm{c}$ & $5.7 \pm 5.1 \mathrm{a}$ & $21.2 \pm 3 b$ & 67 \\
\hline PM & $140 \pm 60 \mathrm{c}$ & $5.1 \pm 0.3 \mathrm{c}$ & $2.8 \pm 1.2 \mathrm{c}$ & $3.3 \pm 0.8 b$ & $0.9 \pm 0.9 \mathrm{c}$ & $24.3 \pm 2 b$ & 75 \\
\hline PAN & $360 \pm 80 b$ & $16.3 \pm 2.8 b$ & $8.4 \pm 3.2 b$ & $0.3 \pm 0.1 \mathrm{~d}$ & $1.4 \pm 3.2 \mathrm{c}$ & $41.1 \pm 6 \mathrm{a}$ & 87 \\
\hline PA & $0 \mathrm{~d}$ & $0 \mathrm{~d}$ & $3.6 \pm 0.8 \mathrm{c}$ & $6.8 \pm 0.9 \mathrm{a}$ & $4.2 \pm 0.4 b$ & $48.7 \pm 4 \mathrm{a}$ & 100 \\
\hline \multicolumn{8}{|l|}{ ANOVA $^{x}$} \\
\hline NAA & $* *$ & $* *$ & $* *$ & $* *$ & NS & NS & \\
\hline Sucrose & $* *$ & $* *$ & NS & NS & $* *$ & $* *$ & \\
\hline NAA $\times$ sucrose & $* *$ & $* *$ & $* *$ & $* *$ & $* *$ & NS & \\
\hline
\end{tabular}

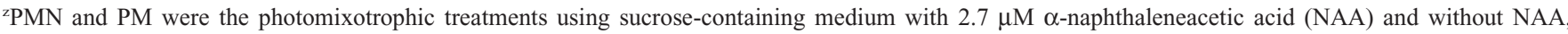
respectively. PAN and PA were the photoautotrophic cultures using sucrose-free medium with $2.7 \mu \mathrm{M}$ NAA and without NAA, respectively.

${ }^{\mathrm{y}}$ Data are mean $\pm \mathrm{SE}$, and treatments denoted by the same letter in a column were not significantly different $(P \leq 0.01)$.

${ }^{\mathrm{x}}$ Analysis of variance was applied to two levels of NAA and sucrose concentrations, respectively.

$\mathrm{DW}=$ dry weight; $\mathrm{NS}=$ nonsignificant

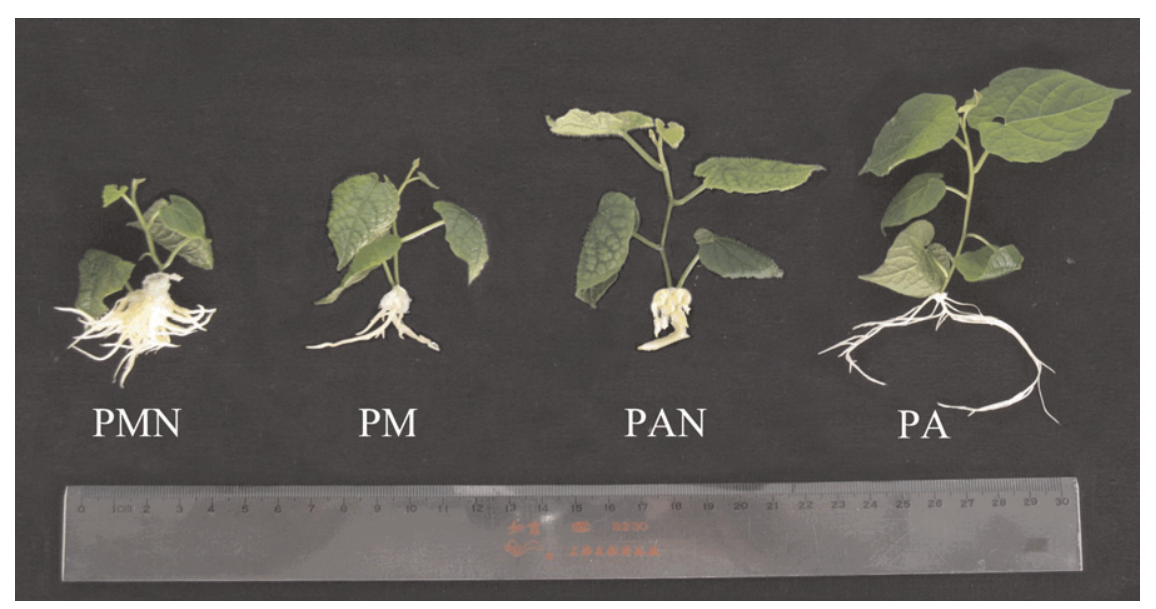

Fig. 5. Effects of sucrose and $\alpha$-naphthaleneacetic acid (NAA) on growth of Momordica grosvenori plantlets on Day 26. PMN and PM were the photomixotrophic cultures using sucrose-containing medium with $2.7 \mu \mathrm{M}$ NAA and without NAA, respectively. PAN and PA were the photoautotrophic cultures using sucrose-free medium with $2.7 \mu \mathrm{M}$ NAA and without NAA, respectively.

been explained by low photosynthetic ability. In the present study, the photosynthetic ability and the growth rate in the control treatment were much lower than those of the PA treatments. The lowest photosynthesis and growth in the control treatment was probably because of a combination of sucrose-containing medium, low light intensity, and infrequent air exchanges in the vessel. Generally, sugar in the culture medium suppresses photosynthesis of plantlets in vitro (Desjardins et al., 1995). Plantlets cultured in sugarcontaining medium depend more on the sucrose and less on $\mathrm{CO}_{2}$ in the air (Kubota and Kozai, 2001). The low photosynthetic ability of plantlets was considered to reduce survival and growth rate during acclimatization (Grout, 1978; Preece and Sutter, 1991). These findings indicate that sugar was not required for the growth of plantlets under high PPFD and $\mathrm{CO}_{2}$ enrichment.

The chlorophyll content of plants plays an important role in the absorption of light during photosynthesis. In the present study, the presence or absence of sucrose in the culture medium significantly influenced chlorophyll content of the plantlets. When the plantlets were grown under the same light intensity $\left(25 \mu \mathrm{mol} \cdot \mathrm{m}^{-2} \cdot \mathrm{s}^{-1}\right)$, the presence of sucrose in the medium reduced the chlorophyll content and lowered the photosynthetic ability in the control treatment (Fig. 3). In contrast, sucrose-free cultures produced more chlorophyll and increased the photosynthetic ability in $\mathrm{L}_{25}$ treatment. This demonstrates that PA could improve photosynthetic ability of plantlets by stimulating chlorophyll synthesis and thus in turn improves adaptation to low light conditions.

The light intensity also significantly affected the chlorophyll content in the PA plantlets. The chlorophyll content increased as the light intensity rose from 25 to 50 $\mu \mathrm{mol} \cdot \mathrm{m}^{-2} \cdot \mathrm{s}^{-1}$ but decreased at higher intensities, 50 to $200 \mu \mathrm{mol} \cdot \mathrm{m}^{-2} \cdot \mathrm{s}^{-1}$. This suggested that the plants could balance light absorption and translation by regulating chlorophyll synthesis (Bailey et al., 2001). The change of chlorophyll content in our study was possibly an acclimation of the plantlets to different light intensity.

Acclimation of the plantlets to light intensity. For many plants, changes in light intensity may elicit physiological responses at the level of leaf and chloroplast (Bailey et al., 2001; Königer et al., 2008). In the present study, leaves responded to light intensity by adjusting the chlorophyll content. Chloroplast responded to light intensity by changing chlorophyll $\mathrm{a} / \mathrm{b}$ and adjusting to the ETR. As shown in Figures 3 and 4, an increase in chlorophyll $\mathrm{a} / \mathrm{b}$ accompanied an increase in the ETR, except when the second leaf from the $\mathrm{L}_{200}$ treatment was sampled (Fig. 4A). An increase in chlorophyll $a / b$ was associated with a decrease in the size of the PSII lightharvesting antenna (Bailey et al., 2001; Leong and Anderson, 1984; Masuda et al., 2002). Moreover, a faster ETR was associated with an increase in the reaction-center content of PSII (Evans, 1987; Leong and Anderson, 1984). Bailey et al. (2001) and Königer et al. (2008) also found changes in the numbers of chloroplasts and leaf thickness associated with changes in chlorophyll content and photosynthetic capacity in Arabidopsis thaliana grown under different light intensities.

Photoinhibition may occur when light intensity exceeds what is required for the saturation of photosynthesis. This adverse phenomenon can manifest as a loss of PSII activity (Masuda et al., 2002). This could account for the lower capacity of ETR as shown in the second leaf from the $\mathrm{L}_{200}$ treatment in this study (Fig. 4A). In photoinhibition, the chloroplasts contained much of the same amount of PSII as they did under low light. However, up to $\approx 80 \%$ of PSII was photochemically inactive because of photodamage (Vasilikiotis and Melis, 1994). During the growth of plantlets, the upper leaves may create a dense canopy that protected the lower ones from photodamage. Thus, photodamage of PSII reaction centers could be repaired and chlorophyll antennae enlarged (Masuda et al., 2002; Neidhardt et al., 1998; Webb and Melis, 1995). Perhaps this is why, in the $\mathrm{L}_{200}$ plantlets, the second and third leaves from the apex had the lowest and highest ETRs, respectively (Fig. 4). The third leaves had the highest ETRs, which contributed to the $\mathrm{L}_{200}$ plantlets having the highest dry weight. The plantlet growth in the $\mathrm{L}_{200}$ treatment, however, was not as good as that in the $\mathrm{L}_{100}$ treatment because of the stunted plantlets with aging stems and small and yellow leaves. The formation of lateral branches in the $\mathrm{L}_{200}$ treatment may have been caused by high light intensity, which could have inhibited the synthesis of IAA in the shoot apex and apical dominance. 
Plantlet quality and survival percentage. The intrinsic quality of plants produced in vitro was one of the key factors governing the survival percentage during acclimatization to greenhouse or field conditions (AfreenZobayed et al., 1999). The results of our study indicated that micropropagation methods affected plantlet quality. The PA-grown plantlets were of good quality and had a high survival percentage, whereas those grown by PM were of poor quality and few survived transplantation. The effectiveness of a micropropagation system can be measured by the survival percentage of plantlets successfully transferred from culture vessels to a greenhouse or field (Kirdmanee et al., 1995; Xiao and Kozai, 2006). The problems associated with conventional micropropagation of $M$. grosvenori may be primarily a result of the poor-quality plantlets in vitro.

Poor-quality plantlets influence survival and growth in many ways. First, large callus masses in the root system can reduce the uptake or absorption of water and nutrients into the plantlet and cause root decay, resulting in a low survival percentage. Nguyen et al. (1999) suggested that callus at the shoot base of coffee plantlets grown by the conventional method may affect the survival percentage of plantlets when transferred from in vitro to ex vitro. Second, poor plantlets usually grow slowly during ex vitro acclimatization. An extended early period of acclimatization shortens growth in the middle and later stages. This not only contributes to nonbearing or low yields of fruits, but also to little dry matter accumulation. Third, the improper use of plant growth regulators also causes poor growth and nonbearing or low yields of fruits. Fourth, plantlets grown photomixotrophically usually have low photosynthetic ability, and this causes the low survival and growth rate in the acclimatization stage (Grout, 1978). The finding that the enhanced net photosynthetic rate of eucalyptus plantlets in vitro increased the survival percentage ex vitro supports this conclusion (Kirdmanee et al., 1995).

\section{Conclusion}

In comparison with the PM plantlets grown on sucrose- and hormone-containing medium, the PA plantlets grown on sucroseand hormone-free medium had a more developed rooting system, better shoots, higher chlorophyll content, greater photosynthetic capability, and high survival ex vitro. Therefore, PA is an alternative way to produce high-quality $M$. grosvenori plantlets. A $P P F D$ of $100 \mu \mathrm{mol} \cdot \mathrm{m}^{-2} \cdot \mathrm{s}^{-1}$ was more suitable for growth of $M$. grosvenori plantlets. The callus-on-plantlet base reduced viability ex vitro. Callus formation was not only caused by NAA, but also sucrose. Plantlets grown on sucrose- and hormone-free medium had no callus on the roots.

\section{Literature Cited}

Afreen-Zobayed, F., S.M.A. Zobayed, C. Kubota,

T. Kozai, and O. Hasegawa. 1999. Supporting material affects the growth and development of in vitro sweet potato plantlets cultured photoautotrophically. In Vitro Cell. Dev. Bio. Plant. 35:470-474.

Aitken-Christie, J., T. Kozai, and S. Takayama 1995. Automation in plant tissue culture. General introduction and overview, p. 1-18. In: Aitken-Christie, J., T. Kozai, and M.A.L. Smith (eds.). Automation and environmental control in plant tissue culture. Kluwer Academic Publishers, Dordrecht, The Netherlands.

Anderson, J.M., W.S. Chow, and Y.-I. Park. 1995 The grand design of photosynthesis: Acclimatization of the photosynthetic apparatus to environmental cues. Photosynth. Res. 46:129-139.

Bailey, S., R.G. Walters, and S.J.P. Horton. 2001 Acclimatization of Arabidopsis thaliana to the light environment: The existence of separate low light and high light responses. Planta 213:794-801.

Behrenfeld, M.J., O. Prasil, M. Babin, and F. Bruyant. 2004. In search of a physiological basis for covariations in light-limited and lightsaturated photosynthesis. J. Phycol. 40:4-25.

Bolhar-Nordenkampf, H.R., S.P. Long, N.R. Baker, G. Oquist, U. Schreiber, and E.G. Lechner. 1989. Chlorophyll fluorescence as a probe of the photosynthetic competence of leaves in the field: A review of current instrumentation. Funct. Ecol. 3:497-514.

Cheng, G.R. 1987. The survey study on the sweet components of Luohanguo. [in Chinese] Guihaia 7:285-286.

Desjardins, Y., C. Hdider, and J. Riek. 1995. Carbon nutrient in vitro regulation and manipulation of carbon assimilation in micropropagation system, p. 441-465. In: Aitken-Christie J., Kozai T., and M.A.L. Smith (eds.). Automation and environmental control in plant tissue culture. Kluwer Academic Publishers, Dordrecht, The Netherlands.

Evans, J.R. 1987. The relationship between electron transport components and photosynthetic capacity in pea leaves grown at different irradiance. Aust. J. Plant Physiol. 14:157-170.

Fujiwara, K., T. Kozai, and I. Watanabe. 1988. Development of a photoautotrophic tissue culture system for shoots and/or plantlets at rooting and acclimatization stages. Acta Hort. 230:153-158.

Grout, B.W. 1978. Transplanting of cauliflower plants regenerated from meristem culture. II. Carbon dioxide fixation and the development of photosynthetic ability. Hort. Res. 17:65-71.

Hirashima, M., S. Satoh, R. Tanaka, and A Tanaka. 2006. Pigment shuffling in antenna systems achieved by expressing prokaryotic chlorophyllide a oxygenase in Arabidopsis. J. Biol. Chem. 281:15385-15393.

Kirdmanee, C., Y. Kitaya, and T. Kozai. 1995. Effect of $\mathrm{CO} 2$ enrichment and supporting material in vitro on photoautotrophic growth of Eucalyptus plantlets in vitro and ex vitro. In Vitro Cell. Dev. Bio. Plant. 31:144-149.

Kitaya, Y., O. Fukuda, T. Kozai, and C. Kirdmanee. 1995. Effect of light intensity and lighting direction on the photoautotrophic growth and morphology of potato plantlets in vitro. Sci. Hort. 62:15-24.

Königer, M., J.A. Delamaide, E.D. Marlow, and G.C. Harris. 2008. Arabidopsis thaliana leaves with altered chloroplast numbers and chloroplast movement exhibit impaired adjustments to both low and high light. J. Expt. Bot. 59:2285-2297.

Kozai, T. 1991. Photoautotrophic micropropagation. In Vitro Cell. Dev. Bio. Plant. 27:47-51.

Kozai, T., K. Fujiwara, and I. Watanabe. 1986. Fundamental studies on environments in plant tissue culture vessels (2): Effects of stoppers and vessels on gas exchange rates between inside and outside of vessels closed with stoppers. J. Agr. Met. 42:119-127.

Kozai, T., H. Oki, and K. Fujiwara. 1990. Photosynthetic characteristics of cymbidium plantlet in vitro. Plant Cell Tissue Organ Cult. 22:205221.

Kubota, C. and T. Kozai. 2001. Growth and net photosynthetic rate of tomato plantlets during photoautotrophic and photomixotrophic micropropagation. HortScience 36:49-52.

Leong, T.Y. and J.M. Anderson. 1984. Adaptation of thylakoid membranes of pea chloroplasts to light intensities. I. Study on the distribution of chlorophyll-protein complex. Photosynth. Res. 5:105-115.

Lin, W., Q. Li, H. Peng, J. Xue, S. Liang, and Y. Huang. 2003. Problem and solution of tissuecultured seeding cultivation of Momordica grosvenori [in Chinese]. Guang Xi Agricultural Sciences 4:74-75

Lindahl, M., D. Yang, and B. Andersson. 1995. Regulatory proteolysis of the major light harvesting chlorophyll $\mathrm{a} / \mathrm{b}$ binding protein of photosystem II by a light induced membrane associated enzymatic system. Eur. J. Biochem. 231:503-509.

Masuda, T., J.E.W. Polle, and A. Melis. 2002. Biosynthesis and distribution of chlorophyll among the photosystems during recovery of the green alga Dunaliella salina from irradiance stress. Plant Physiol. 128:603-614.

Moran, R. 1982. Formulae for determination of chlorophyllous pigments extracted with N, Ndimethylformamide. Plant Physiol. 69:13761381.

Moran, R. and D. Porath. 1980. Chlorophyll determination in intact tissues using N,N-dimethylformamide. Plant Physiol. 65:478-479.

Murashige, T. and F. Skoog. 1962. A revised medium for rapid growth and bioassays with tobacco tissue cultures. Physiol. Plant. 15:473497.

Neidhardt, J., J.R. Benemann, L. Zhang, and A. Melis. 1998. Photosystem II repair and chloroplast recovery from irradiance stress: Relationship between chronic photoinhibition, light-harvesting chlorophyll antenna size and photosynthetic productivity in Dunaliella salina (green algae). Photosynth. Res. 56:175184.

Nguyen, T.Q., T. Kozai, K.L. Nguyen, and U.V. Nguyen. 1999. Effects of sucrose concentration, supporting material and number of air exchanges of the vessel on the growth of in vitro coffee plantlets. Plant Cell Tissue Organ Cult. 58:51-57.

Preece, J.E. and E.G. Sutter. 1991. Acclimatization of micropropagated plants to the greenhouse and field, p. 71-93. In: Debergh, P. and R. Zimmerman (eds.). Micropropagation technology and application. Kluwer Academic Publishers, Dordrecht, The Netherlands.

Ralph, P.J., A. MacMinn, K.G. Ryan, and C. Ashworth. 2005. Short-term effect of temperature on the photokinetics of micro-algae from the surface layers of antarctic ice. J. Phycol. 41:763-769.

Serôdio, J., S. Vieira, S. Cruz, and H. Coelho. 2006. Rapid light-response curves of chlorophyll fluorescence in microalgae: Relationship to steady-state light curves and non-photochemical quenching in benthic diatom-dominated assemblages. Photosynth. Res. 90:29-43.

Serret, M.D., M.I. Trillas, M. Matas, and J.L. Araus. 1996. Development of photoautotrophy and photoinhibition of gardenia jasminoides 
plantlets during micropropagation. Plant Cell Tissue Organ Cult. 45:1-16.

Takasaki, M., T. Konoshima, Y.J. Murata, M. Sugiura, H. Nishino, H. Tokuda, K. Matsumoto, R. Kasai, and K. Yamasaki. 2003. Anticarcinogenic activity of natural sweeteners, cucurbitane glycosides, from Momordica grosvenori. Cancer Lett. 198:37-42.

Vasilikiotis, C. and A. Melis. 1994. Photosystem II reaction center damage and repair cycle: Chloroplast acclimatization strategy to irradiance stress. Proc. Natl. Acad. Sci. USA 91:72227226.
Webb, M.R. and A. Melis. 1995. Chloroplast response in Dunaliella salina to irradiance stress: Effect on thylakoid membrane protein assembly and function. Plant Physiol. 107:885-893.

Xiao, Y. and T. Kozai. 2004. Commercial application of a photoautotrophic micropropagation system using large vessels with forced ventilation: Plantlets growth and production cost. HortScience 39:1387-1391.

Xiao, Y. and T. Kozai. 2006. Photoautotrophic growth and net photosynthetic rate of sweet potato plantlets in vitro as affected by the number of air exchanges of the vessel and type of supporting material. Tsinghua Sci. Technol. 11:481-489.

Xu, H., F. Zhou, B. He, T. Lan, N. Yu, L. Chen, and B. Sha. 2006. Different bio-effects of LFS, KT and $\mathrm{N}^{6}$-BA on the tissue culture of Siraitia Grosvenori (Swingle) C. Jeffrey in vitro [in Chinese]. Seed 25:4-9.

Yao, J., H. Tang, L. Zhou, W. Shen, Y. Li, X. Tian, and X. Ding. 2008. Effect of Momordica grosvenori extracts on the mouse exercise capacity and the hepatic tissue antioxidant injury [in Chinese]. Chin J Sports Med 27:2212-2223. 